\title{
Control of Electron Localization in Deuterium Molecular Ions using an Attosecond Pulse Train and a Many-Cycle Infrared Pulse
}

\author{
K. P. Singh, ${ }^{1, *}$ F. He, ${ }^{1}$ P. Ranitovic,,${ }^{1,2,3}$ W. Cao, ${ }^{1}$ S. De,${ }^{1}$ D. Ray,${ }^{1}$ S. Chen, ${ }^{1}$ U. Thumm, ${ }^{1}$ A. Becker, ${ }^{2}$ M. M. Murnane, ${ }^{2}$ \\ H. C. Kapteyn, ${ }^{2}$ I. V. Litvinyuk, ${ }^{1}$ and C. L. Cocke ${ }^{1}$ \\ ${ }^{1}$ J. R. Macdonald Laboratory, Physics Department, Kansas State University, Manhattan, Kansas 66506, USA \\ ${ }^{2}$ JILA and Department of Physics, University of Colorado at Boulder, Boulder, Colorado 80309-0440, USA \\ ${ }^{3}$ Department of Physics, Stockholm University, SE 10691, Stockholm, Sweden
}

\begin{abstract}
We demonstrate an experimental control of electron localization in deuterium molecular ions created and dissociated by the combined action of an attosecond pulse train and a many-cycle infrared (IR) pulse. The attosecond pulse train is synthesized using both even and odd high order harmonics of the driving IR frequency so that it can strobe the IR field once per IR cycle. An asymmetric ejection of the deuterium ions oscillates with the full IR period when the APT-IR time-delay is scanned. The observed control is due to the creation of a coherent superposition of $1 s \sigma_{g}$ and $2 p \sigma_{u}$ states via interference between one-photon and two-photon dissociation channels.
\end{abstract}

The control of electron dynamics and localization in molecules on the attosecond (as) time-scale is crucial in order to steer many physical and chemical processes [1-3]. A key step forward in this direction was achieved by an experimental demonstration of the electron localization in the dissociating molecular ion $\left(\mathrm{D}_{2}^{+}\right)$using few-cycle, carrier-envelope phase stabilized, IR pulses [4]. By varying the phase of the carrier envelope of the intense IR pulse, the driven electron can be localized on one or the other nucleus, an effect which is manifested by the asymmetric ejection of produced ionic fragments in a preferential direction. This asymmetric ion ejection happens due to the creation of a coherent superposition of $1 s \sigma_{g}$ and $2 p \sigma_{u}$ states during the dissociation of $\mathrm{D}_{2}^{+}$. Depending upon their relative phase, which is controlled by tuning the phase of the carrier envelop of the IR pulse, the localization of the electron on one of the nuclei takes place selectively. Since the pioneering experiment by Kling et al. [4] using few-cycle pulses, several theoretical approaches have been proposed to achieve as well as explain the electron localization in molecules [5-10]. However, only a few experimental tools exist to date.

On the other hand, the combined action of an attosecond pulse train (APT) and an IR pulse has been a powerful technique in attosecond physics [2]. The APT is usually synthesized by means of the high harmonic generation process, which produces multiple high harmonics of the fundamental driving field [11]. When the spectral phases of these harmonics are locked to each other, their combination can produce attosecond bursts of extreme ultraviolet (XUV) radiation in synchronization with the IR field [1,2]. The APT-IR combination has been employed to observe and control physical processes on a subatomic time-scale, for example, in attosecond spectroscopy [12], in steering attosecond electron wave packets by light [13], in atto- second streak cameras [14], and in controlling photoionization on an attosecond time scale [15]. Despite its great potential, the APT-IR technique has not been used to control the electron localization in molecules yet. This is mainly because one needs a nontrivial APT with one attosecond burst per IR cycle to observe the effect [7], which could only be synthesized recently [16-18].

Here we experimentally demonstrate a new approach to control the electron localization in $\mathrm{D}_{2}$ using the combined action of an APT and a many-cycle IR pulse. The APT is synthesized from both the even and odd harmonics of the driving IR pulse using a two-color technique, resulting in one attosecond pulse per IR cycle [16,17]. With such an APT a higher degree of control is expected since each electron wave packet is directed along the same direction along the internuclear axis [7]. Our technique offers several advantages: (i) it does not need any carrier-envelope phase stabilized pulses, (ii) it works well with routinely available pulse of many cycles, and (iii) it can be applied to other molecular systems by tuning the XUV spectrum appropriately. Our experimental as well as theoretical results demonstrate that the observed control takes place in the low energy dissociation channels of $\mathrm{D}_{2}^{+}$which were not observed so far.

Our experimental setup consists of a Mach Zehnder interferometer for the XUV and IR pulses. The APT is generated in one of its arms by combining a $40 \mathrm{fs}, 800 \mathrm{~nm}$ pulses having an intensity, $I \approx 5.0 \times 10^{13} \mathrm{~W} / \mathrm{cm}^{2}$ with its second harmonic (blue) pulse [16-18]. The blue pulse is produced using a $250 \mu \mathrm{m} \mathrm{BaB} \mathrm{O}_{4}$ crystal with about $20 \%$ efficiency. The IR and blue pulses are overlapped temporally and their polarizations are made parallel [17]. The relative phase between IR and blue pulse is experimentally chosen so as to optimize the yield of even order harmonics. Both the pulses are focused inside a $3 \mathrm{~cm}$ long capillary 
(150 $\mu \mathrm{m}$ inner diameter) filled with Argon gas at 35 Torr. Moreover, because the blue pulse is inherently locked to the IR pulse, the carrier-envelope phase stabilization is no longer needed. The XUV generated by the two-color driving field is spectrally filtered by a $200 \mathrm{~nm}$ thick Al-foil, which cuts off the high harmonics below 11th order along with the residual two-color field. A time-delayed replica of the fundamental IR pulse (probe IR) is recombined collinearly with the XUV, both beams are then focused into an effusive gas jet [see inset in Fig. 1(b)]. The estimated peak IR intensity at the focus is $I \approx 10^{13} \mathrm{~W} / \mathrm{cm}^{2}$ and its focal spot size is much bigger than the XUV focal spot. The produced ionic fragments are detected using a standard cold-target recoil-ion momentum spectroscopy arrangement (COLTRIMS) [19,20] with extraction field of about $2.3 \mathrm{~V} / \mathrm{cm}$.

In order to characterize our XUV, we show in Fig. 1(a) an energy spectrum of the photoelectrons generated by ionizing Ar with the XUV only. The photoelectron spec-
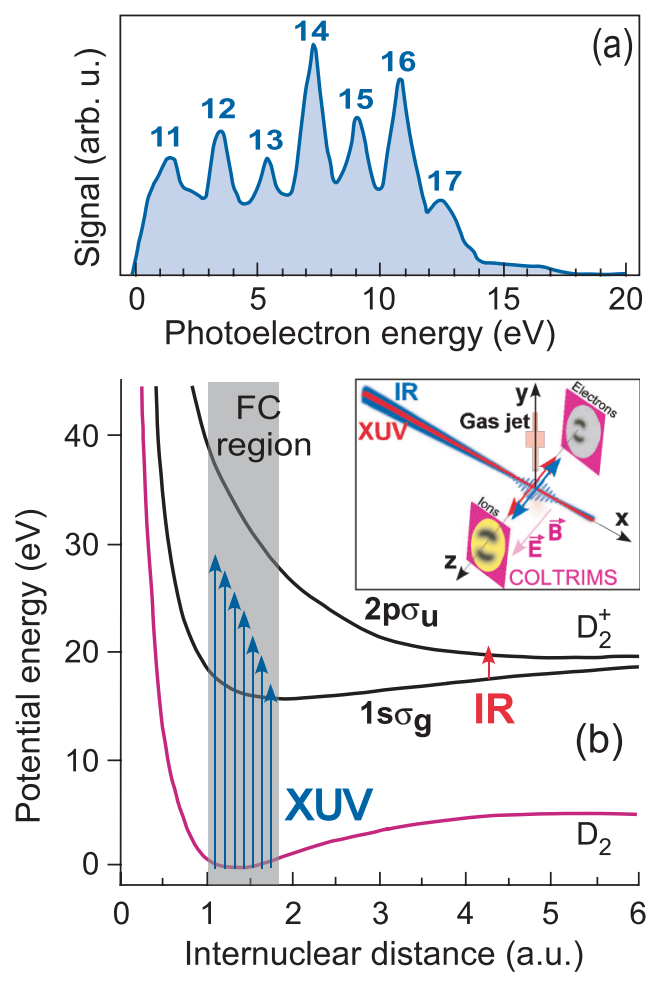

FIG. 1 (color online). The XUV characterization and schematic of the control of electron localization in $\mathrm{D}_{2}$. (a) Photoelectron energy spectrum of Ar produced by the Ar harmonics using the two-color ( $800 \mathrm{~nm}$ and $400 \mathrm{~nm}$ ) driving field. (b) The XUV photon energies from 11th to 17th harmonics (blue arrows) are superimposed on $\mathrm{D}_{2}$ potential curves within the FanckCondon (FC) transition region. The red arrow denote the IRassisted coupling between the $1 s \sigma_{g}$ and $2 p \sigma_{u}$ states (in the united-atom designation) of $\mathrm{D}_{2}^{+}$. Inset: A geometrical sketch of the setup showing the XUV-IR propagation along the $x$ axis, their polarization vectors and the time-of-flight (TOF) direction along the $z$ axis, and the gas jet along the $y$ axis. trum contains well resolved peaks containing both the even and odd harmonics from 11th to 17th order. Similar features have been observed experimentally using two-color driving field and it was even possible to tune the ratio of even to odd harmonics [17]. This frequency spectrum corresponds to a train of attosecond pulses with one attosecond pulse per IR cycle in the time-domain. The transform-limited envelope of the APT is estimated to be about $10 \mathrm{fs}$ long with duration of individual XUV bursts of about 350 as [18]. It has been recently suggested theoretically that the above generated APT is necessary to control the electron localization, because it can strobe the IR field once per optical cycle at its fixed phase, that can be chosen by the APT-IR delay [7].

First, we focus on the molecular dynamics of $\mathrm{D}_{2}$ irradiated with the APT only (without the IR). By comparing the XUV photon energies with the $D_{2}$ potential energy curves [see Fig. 1(b)], we can say that $\mathrm{D}_{2}^{+}$can only be produced in its electronic ground state, $1 s \sigma_{g}$. The 11th harmonic directly populates bound vibrational levels, while the other higher harmonics (above $16 \mathrm{eV}$ ) can populate the bound vibrational states of $1 s \sigma_{g}$ potential, with the electron carrying the excess energy. A small fraction of the XUV populates the vibrational continuum which dissociates $\left[\mathrm{D}_{2}^{+}\left(1 s \sigma_{g}\right) \rightarrow \mathrm{D}^{+}+\mathrm{D}\right]$ via the so-called ground state dissociation process [21]. As shown in Fig. 2(a) the time-offlight spectrum shows a dominant of $\mathrm{D}_{2}^{+}$along with a very small peak for the $\mathrm{D}^{+}$ions [see inset in Fig. 2(a)]. A symmetric momentum distribution of the $\mathrm{D}^{+}$ions in the $x z$ plane is observed as shown in Fig. 2(b). Since the APTinduced ground state dissociation takes place only through the $1 s \sigma_{g}$ state, it leads to equal dissociation probability of $\mathrm{D}^{+}$along the "left" or the "right" direction.

In order to demonstrate an experimental control of the electron localization we now overlap the APT with the IR
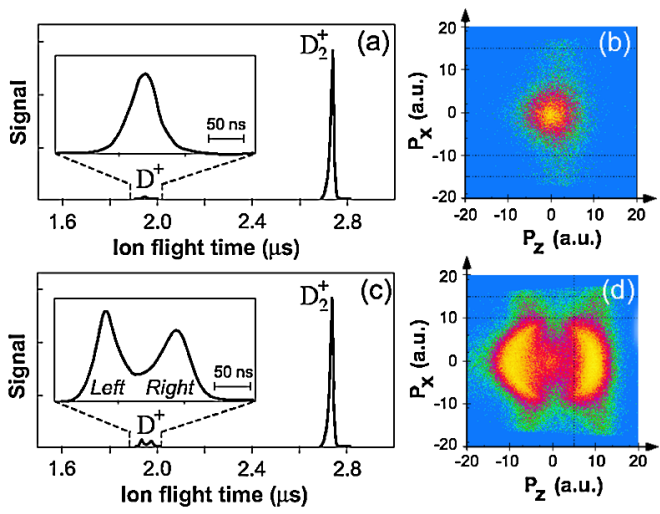

FIG. 2 (color online). Experimental signatures for the directional ejection of $\mathrm{D}^{+}$ions. The ion TOF spectra when $\mathrm{D}_{2}$ is exposed to, (a) XUV only, and (c) XUV with IR. Left and Right denote forward and backward $\mathrm{D}^{+}$ions, respectively. Insets: zoom around the $\mathrm{D}^{+}$TOF. The momentum distribution of the $\mathrm{D}^{+}$ions in the $x z$ plane for the cases, (b) XUV only, and (d) XUV with IR. 
pulse. The IR intensity is weak $\left(I \approx 10^{13} \mathrm{~W} / \mathrm{cm}^{2}\right)$ in order that it cannot ionize $\mathrm{D}_{2}$ by itself. However, the weak IR probe can dissociate $\mathrm{D}_{2}^{+}$ions once they are produced by the APT. An addition of IR on the XUV clearly splits the single peak structure of the $\mathrm{D}^{+}$TOF into a double-peak structure, as shown in Fig. 2(c). This gives us a clear distinction between the $\mathrm{D}^{+}$ions ejected in the left (right) direction, as a result of the localization of electron on the right (left) nucleus. The $x z$ momentum distribution of $\mathrm{D}^{+}$ions in the case of overlapping XUV and IR also shows a double-lobe structure oriented along the polarization vectors of the fields.

The electron localization is quantified via the left-right ejection asymmetry using an asymmetry parameter, $A$, defined as

$$
A=\frac{C_{l}-C_{r}}{C_{l}+C_{r}}
$$

where $C_{l}$ and $C_{r}$ are the total $\mathrm{D}^{+}$counts ejected in the left and right directions, respectively. One can clearly see in Fig. 3 that the experimental asymmetry $A$ oscillates with the period of full IR cycle of $2.7 \mathrm{fs}$. The peak-to-peak contrast of the oscillations is about $5 \%$ for our weak probe IR intensity. It is worth mentioning that similar oscillations in the asymmetry have been observed previously, but for the recollision-induced dissociation of $\mathrm{D}_{2}$ via the $2 p \sigma_{u}$ state [4]. Our results are the first experimental observation of the asymmetry using APT and IR starting from the $1 s \sigma_{g}$ state.

In order to gain a better insight into the mechanism of the experimental control of the electron localization, we solve the time-dependent Schrödinger equation (TDSE) for the coupled $1 s \sigma_{g}$ and $2 p \sigma_{u}$ states of $\mathrm{D}_{2}^{+}[4,10]$. The molecular axis is assumed to be aligned along the laser polarization vector of the IR (XUV) pulse. The APTinduced sudden ionization of $\mathrm{D}_{2}$ is modeled by launching

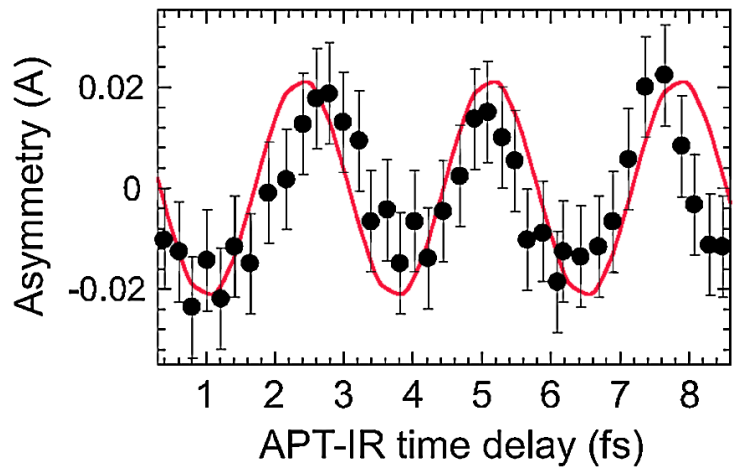

FIG. 3 (color online). The asymmetry parameter $A$ versus the APT-IR time delay. Circles: experimental data, solid line: theory. The data have been smoothened using three-point runningaverage of raw data. The error bars denote the statistical error in total $\mathrm{D}^{+}$counts for the averaged data. The positive delays correspond to the APT followed by the IR pulse. a Franck-Condon (FC) wave packet onto the $1 s \sigma_{g}$ state because the internuclear separation does not change during the photoionization. This FC wave packet then evolves under the time-delayed IR pulse. Note that the FC wave packet is launched only once, unlike in the experiment where it is launched many times due to the periodicity of the APT. Following the standard formulation $[5,6]$, the asymmetry parameter is computed as $A=\left(P_{l}-P_{r}\right) /$ $\left(P_{l}+P_{r}\right)$, where $P_{l}$ and $P_{r}$ are the dissociation probabilities in the left (along positive IR field amplitude) and right direction.

The theoretical asymmetry versus the XUV-IR delay as shown by the solid line in Fig. 3, clearly oscillates with the full IR cycle periodicity; its contrast and periodicity matches closely with the experiment. The oscillations of the theoretical curve, however, do not seem to match the data exactly, which we attribute to a slight drift in the interferometer during the long (several hours) data acquisition. The model used here neglects the interference effects between successively launched wave packets by the APT. Nevertheless, a good agreement with the data suggests that such effects are negligible in the overall asymmetry. Note that in the above comparison both the data and the theory are integrated over all the available ion momenta.

The interaction of the long IR pulse with $\mathrm{D}_{2}^{+}\left(1 s \sigma_{g}\right)$ (created in the IR pulse) couples $1 s \sigma_{g}$ and $2 p \sigma_{u}$ states by driving population between these two levels many times [Fig. 4(a)]. This IR-assisted mixing causes dissociation through the bond-softening and above threshold dissociation channels [22]. The $\mathrm{D}_{2}^{+}$in $1 s \sigma_{g}$ either absorbs one IR photon to dissociates on $2 p \sigma_{u}$, or, it first absorbs three IR photons followed by emission of one-photon to dissociate on $1 s \sigma_{g}$ curve. The interference between these two quan-
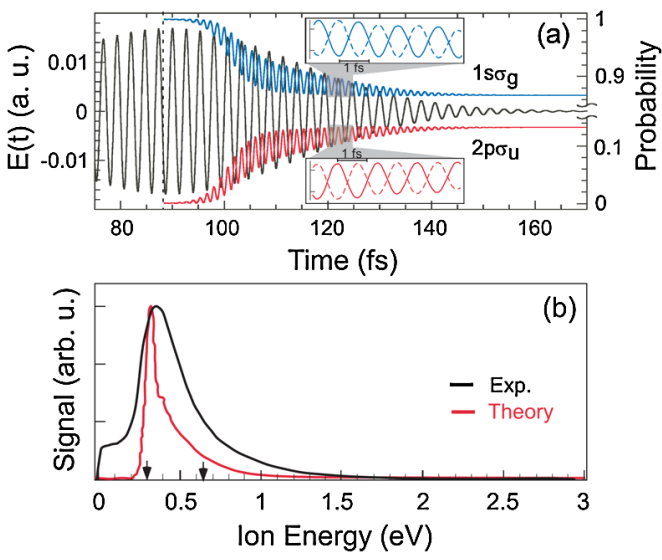

FIG. 4 (color online). The mechanism of the electron localization control. (a) The time evolution of $1 s \sigma_{g}$ and $2 p \sigma_{u}$ populations following the launch of a $\mathrm{FC}$ wave packet for zero delay (dashed line) in the IR pulse $\left(I \approx 10^{13} \mathrm{~W} / \mathrm{cm}^{2}, 40 \mathrm{fs}\right)$. Insets: Zoom on population dynamics for the zero delay and for $0.67 \mathrm{fs}$ delay (dashed curves). (b) Experimental and theoretical $\mathrm{D}^{+}$ion energies integrated over all delays. 
tum paths leads to the creation of a coherent superposition state of $1 s \sigma_{g}$ and $2 p \sigma_{u}$. By scanning the APT-IR timedelay, one can control the relative phase between the coherent superposition states leading to different WP dynamics [see insets in Fig. 4(a)]. Such superposition state when projected onto the electronic basis set corresponding to the left-right localization of electron leads to the observed control of the electron localization. The dissociation energy of $\mathrm{D}^{+}$ions via one-photon and two-photon absorption would be about 0.3 and $0.65 \mathrm{eV}$, respectively, but each process populates a substantial range of ion energies and considerable overlap is expected. Our experimental and theoretical ion energy distribution, integrated over all delays, is shown in Fig. 4(b). This distribution includes contributions from both one-photon and two-photon channels, though one cannot resolve them in the broad distribution. It is essential that both gerade and ungerade channels be populated for the same ion energy for asymmetry to be produced. It is worth mentioning that the twophoton channel is much weaker than the one-photon one (much smaller population in $2 p \sigma_{u}$ state in Fig. 4(a)], because of the weak IR intensity. Therefore, the interference between these two channels produces rather low contrast in the asymmetry oscillations. Nevertheless, our results demonstrate the control of the electron localization using APT and IR pulses for the first time in the low energy $(<1 \mathrm{eV})$ dissociation channel. The initial step here is provided by the photoexcitation by the XUV radiation to the $1 s \sigma_{g}$ curve, whereas in Ref. [4] this step was caused by electron recollision, exciting the $2 p \sigma_{u}$ state, and generating the asymmetry in the high ion region.

As a supplement to the detailed model result, we offer the following simple physical picture of the cause of the observed oscillation. The APT launches a FC wave packet on the $1 s \sigma_{g}$ curve. The wave function of the bound electron at this point is symmetrically distributed between the nuclei, but as time proceeds, the IR field causes this wave function to oscillate between the charge centers (nuclei). As the centers separate, at some localization distance (which depends on the IR strength somewhat, but is around 4-6 a.u.) this wave function is "trapped" by one or the other center. As one varies the IR phase at which this oscillation starts (i.e., the launch time of the FC wave packet), one correspondingly varies the phase of the oscillation at which the electron motion is trapped, and thus the asymmetry.

In conclusion, we have demonstrated a robust experimental tool to control the electron localization in a molecule using APT and many-cycle IR pulses. The APT is synthesized using both the even and odd higher order harmonics so that it contains one attosecond pulse per IR cycle. By varying the APT-IR time-delay around their time-overlap, the directional asymmetry of the $\mathrm{D}^{+}$ejection exhibits oscillations with the full IR period. The observed control is due to the IR-assisted creation of a coherent superposition of $1 s \sigma_{g}$ and $2 p \sigma_{u}$ states during dissociation of $\mathrm{D}_{2}^{+}$, following its production in $1 s \sigma_{g}$ by the APT. The experimental results are in agreement with the simulations based on coupled-state TDSE for $\mathrm{D}_{2}^{+}$. Our setup offers a unique opportunity to perform a kinematically complete localization experiment by collecting the ionic fragment $\left(\mathrm{D}_{2}^{+}, \mathrm{D}^{+}\right)$in coincidence with the ionized electron, that could provide a rigorous insight into the localization mechanism. Because of the tunability of the XUV spectrum [23,24], the APT-IR technique is potentially attractive to explore similar effects in other molecules $(\mathrm{CO}, \mathrm{NO}$, etc.), as well as in isolating different possible control mechanisms in a given molecule.

This work was supported by the Chemical Sciences, Geosciences and Biosciences Division, Office of Basic Energy Sciences, the U.S. Department of Energy, the U.S. Army Research Office under Grant No. W911NF07-1-0475, and NSF Grant No. 0822646.

*Present address: Department of Physics, Indian Institute of Science Education and Research Mohali, Chandigarh, India.

[1] P. B. Corkum and F. Krausz, Nature Phys. 3, 381 (2007).

[2] F. Krausz and M. Ivanov, Rev. Mod. Phys. 81, 163 (2009).

[3] A. D. Baudrauk et al., Int. J. Quantum Chem. 100, 834 (2004).

[4] M. F. Kling et al., Science 312, 246 (2006).

[5] F. He, C. Ruiz, and A. Becker, Phys. Rev. Lett. 99, 083002 (2007).

[6] F. He, A. Becker, and U. Thumm, Phys. Rev. Lett. 101, 213002 (2008).

[7] F. He, C. Ruiz, and A. Becker, J. Phys. B 41, 081003 (2008).

[8] A. Staudte et al., Phys. Rev. Lett. 98, 073003 (2007).

[9] S. Gräfe and M. Y. Ivanov, Phys. Rev. Lett. 99, 163603 (2007).

[10] X. M. Tong and C. D. Lin, Phys. Rev. Lett. 98, 123002 (2007).

[11] P. Agostini and L. F. DiMauro, Rep. Prog. Phys. 67, 813 (2004).

[12] P. H. Bucksbaum, Science 317, 766 (2007).

[13] R. Kienberger et al., Science 297, 1144 (2002).

[14] J. Itatani et al., Phys. Rev. Lett. 88, 173903 (2002).

[15] P. Johnsson et al., Phys. Rev. Lett. 99, 233001 (2007).

[16] J. Mauritsson et al., Phys. Rev. Lett. 97, 013001 (2006).

[17] Dudovitch et al., Nature Phys. 2, 781 (2006).

[18] E. Mansten et al., New J. Phys. 10, 083041 (2008).

[19] J. Ullrich et al., Rep. Prog. Phys. 66, 1463 (2003).

[20] R. Dorner et al., Phys. Rep. 330, 95 (2000).

[21] I. Ben-Itzhak et al., Phys. Rev. Lett. 85, 58 (2000).

[22] A. Zavriyev et al., Phys. Rev. Lett. 70, 1077 (1993).

[23] A. Rundquist et al., Science 280, 1412 (1998).

[24] F. Brandi et al., Phys. Rev. Lett. 91, 163901 (2003). 